\title{
Panorama da Pesquisa Acadêmica Brasileira em Nanocompósitos Polímero/Argila e Tendências para o Futuro
}

\author{
Priscila Anadão, Hélio Wiebeck, Francisco R. Valenzuela-Díaz \\ Engenharia Metalúrgica e de Materiais, Escola Politécnica, USP
}

\begin{abstract}
Resumo: A pesquisa em nanocompósitos polímero/argila foi iniciada pela Toyota no final dos anos 80. A partir deste marco, a comunidade científica mundial voltou sua atenção para este tipo de material graças às propriedades mecânicas, químicas e térmicas avançadas em comparação com os compósitos tradicionais. Não foi diferente no Brasil, tal atenção pode ser comprovada pelos inúmeros trabalhos científicos sobre nanocompósitos que começaram a despontar no início do século XXI. Assim, o presente artigo objetiva, após discorrer sobre as definições que concernem nanocompósitos polímero/argila, apresentar as pesquisas científicas brasileiras em nanocompósitos de polímeros de uso geral, de polímeros de engenharia e de outros tipos de polímeros, relacionando-os com o método de produção empregado, morfologia final e propriedades decorrentes da formação de nanocompósitos. Por fim, o mapa da pesquisa brasileira em nanocompósitos polímero/argila e sua perspectiva para o futuro são apresentados.
\end{abstract}

Palavras-chave: Nanocompósito, polímero, argila.

\section{Scenario of the Brazilian Academic Research into Nanocomposites Polymer/Clay and Trends for the Future}

\begin{abstract}
The research on polymer/clay nanocomposites was initiated by Toyota in the late 80s. From then on, the world scientific community has turned its attention to this type of material due to the enhanced mechanical, chemical and thermal properties in comparison to traditional composites. In Brazil, it was not different, as can be seen from the countless scientific works on nanocomposites which have emerged in the early $21^{\text {th }}$ century. The aim thus is, after discussing the definitions related to the polymer/ clay nanocomposites, to show Brazilian scientific works on the general use of plastics, engineering plastics and other types of polymers, correlating them with their production method, final morphology and resulting properties. Finally, a map of the Brazilian research into polymer/clay nanocomposites
\end{abstract} and their future perspective are presented.

Keywords: Nanocomposite, polymer, clay.

\section{Introdução}

Nanocompósitos polímero/argila são uma nova classe de compósitos com matrizes poliméricas onde a fase dispersa é o silicato, constituída por partículas elementares que têm pelo menos uma de suas dimensões da ordem de nanômetros. As partículas minerais mais utilizadas nestes nanocompósitos são as argilas esmectíticas (montmorilonita, saponita e hectorita), tendo as suas partículas morfologia lamelar, com lados da ordem de um micrômetro e espessura de aproximadamente um nanômetro ${ }^{[1]}$.

A camada unitária da montmorilonita (MMT) consiste em três folhas: uma folha octaédrica de hidrargilita-brucita incluídas entre duas folhas tetraédricas de silício e oxigênio (Figura 1). A água e outras moléculas, tais como moléculas orgânicas, podem entrar nas camadas unitárias causando a sua expansão. A distância entre camadas da montmorilonita varia de 9,6 $\AA$, quando nenhuma molécula está entre as camadas unitárias, até quase a separação completa em alguns $\operatorname{casos}^{[2]}$.

O interesse na produção de nanocompósitos poliméricos advém da larga gama de propriedades resultantes, como, maior resistência mecânica, térmica e à luz ultravioleta (UV), alta impermeabilidade a gases, baixa expansibilidade e flexibilidade de processamento ${ }^{[3]}$.

Diversas revisões internacionais sobre nanocompósitos polímero/ argila foram publicadas nos últimos anos, dentre elas, podemos citar as de autoria de $\mathrm{Mittal}^{[4]}$, Choudalakis e Gotsis ${ }^{[5]}$, Yeh e Chang ${ }^{[6]}$, Pavlidou e Papaspyrides ${ }^{[7]}$, Ray e Okamoto ${ }^{[8]}$, Schmidt, Shah e Giannelis ${ }^{[9]}$ e Alexandre e Dubois ${ }^{[1]}$.
Considerando então a grande atenção que vem sendo dada a este tipo de material, torna-se oportuna a apresentação do panorama da pesquisa acadêmica brasileira na contribuição ao desenvolvimento desta área. Para tal, foi realizada uma amostragem de trabalhos relacionados ao preparo e caracterização destes nanocompósitos, priorizando-se os artigos publicados em revistas nacionais e internacionais até o início de 2010. Sendo assim, muitos trabalhos não serão citados, mas são igualmente relevantes e importantes na contribuição para a consolidação desta importante área de pesquisa no Brasil, cuja velocidade de desenvolvimento está em ascensão.

\section{Breve Histórico dos Nanocompósitos}

Durante e após as crises de 1973 e 1979, o aumento do preço do petróleo era sucessivo, por isso, para redução de custos do material final, empregavam-se minerais, com função de cargas de enchimento, na fabricação de produtos poliméricos ${ }^{[10]}$. $O$ desenvolvimento de compósitos com mica, esferas e fibras de vidro, talco e carbonato de cálcio e outros incentivaram a pesquisa de como estas cargas interagiam com os polímeros.

Os compósitos de argilas e polímeros apareceram bem antes dos anos $80^{[11]}$, mas, somente no final desta década, trabalhos de nanocompósitos termoplásticos foram realizados na Toyota, com poliamida 6 e argilas organofílicas para a fabricação de correias dentadas para automotores. Este material continha 5\% em massa 
de argila, que melhorava sensivelmente as características deste material quando comparadas às da resina poliamida 6 , havendo um aumento de $40 \%$ na tensão de ruptura, $68 \%$ no módulo de tração e $126 \%$ no módulo de flexão, aliado ao aumento na temperatura de distorção térmica (HDT) de $65^{\circ} \mathrm{C}$ para $152^{\circ} \mathrm{C}^{[12]}$.

Assim, as companhias iniciaram a produção dos nanocompósitos termoplásticos, a maioria para uso automotivo, obtendo volume suficiente para a viabilização destes materiais; como exemplo, a General Motors que, em 2003, utilizou $245 \mathrm{t}^{[13]}$. Outra aplicação em destaque dos nanocompósitos é a de aumento de barreira a gases, para a qual se espera um aumento expressivo em futuro próximo ${ }^{[14]}$.

\section{Morfologia}

Dependendo das forças das interações interfaciais entre a matriz polimérica e o silicato (modificado ou não), três tipos diferentes de nanocompósitos são termodinamicamente aceitáveis (Figura 2):

- nanocompósitos intercalados: a inserção da matriz polimérica na estrutura do silicato ocorre de forma regular cristalograficamente, alternando camadas entre argila e polímero, cuja distância entre elas varia de 1 a $4 \mathrm{~nm}$, distância da ordem do comprimento da cadeia polimérica;

- nanocompósitos floculados: conceitualmente seria o mesmo que os nanocompósitos intercalados, porém, as camadas de silicato estão, algumas vezes, floculadas devido à interação entre os grupos hidroxilas do silicato;

- nanocompósitos esfoliados (delaminados): as camadas individuais da argila são randomicamente separadas em uma matriz polimérica contínua em uma distância média que depende da carga da argila.

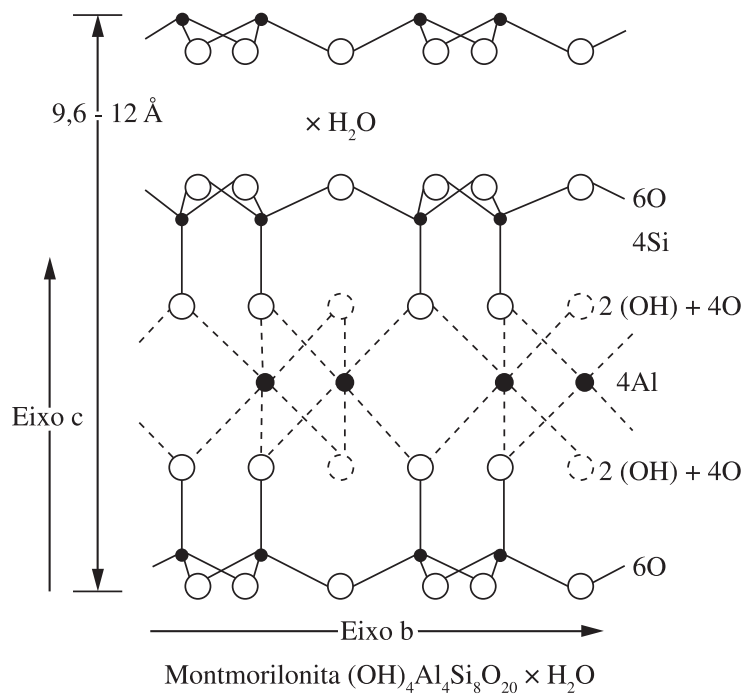

Figura 1. Representação esquemática da estrutura da montmorilonita ${ }^{[2]}$.

\section{Métodos de Produção dos Nanocompósitos}

Quatro são os métodos utilizados no preparo de nanocompósitos. O primeiro deles é a polimerização in situ, no qual se utiliza um monômero como meio para dispersão da argila e condições favoráveis são impostas de modo a realizar a polimerização entre as camadas da argila. A polimerização pode ser iniciada por calor ou radiação, pela difusão de um iniciador adequado ou catalisador fixo dentro da camada, antes do passo de preenchimento por monômero. Pelo fato de a argila possuir alta energia de superfície, ela exerce atração pelas unidades monoméricas para dentro de suas galerias até atingir-se equilíbrio e as reações de polimerização ocorrem entre as camadas de menor polaridade das moléculas intercaladas, deslocando o equilíbrio e então, possibilitando a difusão de novas espécies polares entre as camadas para progressivamente esfoliar a argila.

O segundo método é a dispersão por solução. O silicato é esfoliado em camadas únicas usando um solvente no qual o polímero ou pré-polímero é solúvel. Tais camadas de silicato, detentoras de forças fracas que mantém as camadas unidas, podem ser facilmente dispersas em um solvente adequado através do aumento da entropia pela desorganização das camadas que supera a entropia organizacional das lamelas. O polímero é então adsorvido nas camadas delaminadas e quando o solvente é evaporado (ou a mistura é precipitada), as camadas se reúnem, recheadas com o polímero para formar, no melhor caso, uma estrutura multicamadas ordenada, ou seja, de morfologia intercalada.

Ademais, tem-se a intercalação por fusão. Neste método, o silicato é misturado com a matriz polimérica termoplástica em seu estado fundido. Sob estas condições, o polímero é arrastado para o espaço interlamelar, formando um nanocompósito intercalado ou esfoliado. A força motriz no processo de intercalação por fusão é a contribuição entálpica das interações polímero - argila. A vantagem do emprego desta técnica é a abolição do uso de solvente ${ }^{[3,15]}$.

Por fim, um método menos comum também é empregado, no qual, uma pasta de argila é diretamente misturada com o polímero. A idéia desta técnica consiste no aumento do espaçamento basal da argila no primeiro estágio do processo, pela polimerização dos monômeros ou suspensão da argila em água/solventes e então a matriz polimérica é intercalada no espaçamento basal aumentado ${ }^{[16]}$.

\section{Modificações em Polímero e Argila para Formação de Nanocompósito}

Em alguns casos, o polímero ou argila não são compatíveis quimicamente e assim, para a formação do nanocompósito, há a necessidade de modificação química destes materiais. Duas técnicas comumente utilizadas são o emprego de agente compatibilizante e a organomodificação de argila.

Geralmente, um agente compatibilizante pode ser um polímero que ofereça natureza química compatível com o polímero e a argila. Por meio de tratamento, como a graftização (enxertia) de um

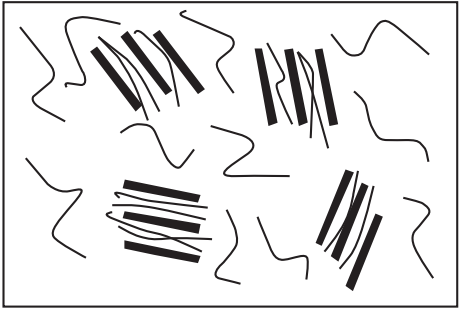

Intercalado

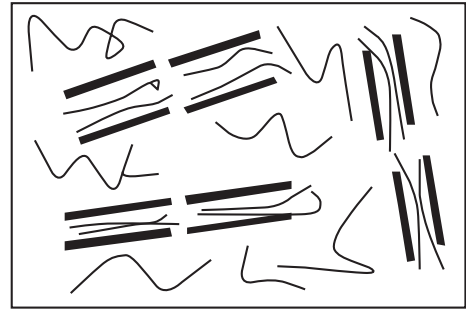

Intercalado e floculado

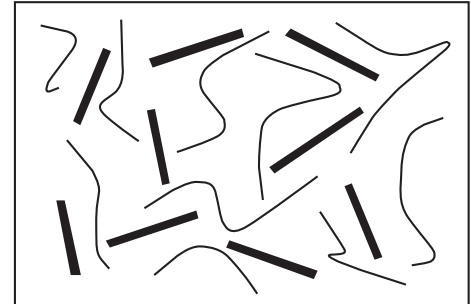

Esfoliado

Figura 2. Ilustração dos três tipos de nanocompósitos termodinamicamente aceitáveis. 
elemento químico contendo grupos reativos ou copolimerização com outro polímero contendo também grupos reativos, proporciona-se compatibilidade entre os materiais que formarão o nanocompósito ${ }^{[3]}$.

Com relação à argila, como poderá ser visto nos tópicos relativos aos diversos tipos de nanocompósitos, a grande maioria deles foi preparada com argilas importadas, embora, haja um número bastante significativo de trabalhos realizados com argilas brasileiras principalmente pelo grupo de pesquisa da Universidade Federal de Campina Grande (UFCG). Para ambas as procedências de argila, é interessante que haja um maior nível de interações entre cadeias poliméricas e camadas de argila, de forma que as propriedades finais dos nanocompósitos sejam melhoradas. É neste contexto que se utiliza, portanto, a técnica de organomodificação das argilas.

Tal método consiste na troca dos cátions interlamelares e da superfície (normalmente os íons sódio e cálcio) por moléculas orgânicas detentoras de carga positiva que continuam a neutralizar as cargas negativas advindas das camadas de silicato, a fim de se introduzir hidrofobicidade e então, produzir-se uma argila organofílica. Nos trabalhos brasileiros, geralmente são empregados cátions surfactantes do tipo alquilamônio de fórmula geral $\left[\left(\mathrm{CH}_{3}\right)_{3} \mathrm{NR}\right]^{+}$ou $\left[\left(\mathrm{CH}_{3}\right)_{2} \mathrm{NRR}^{\prime}\right]^{+}$, onde $\mathrm{R}$ e R' são grupos de hidrocarbonetos de cadeia longa (geralmente derivados de ácidos graxos) ligados diretamente a um átomo de nitrogênio detentor da carga positiva da molécula ${ }^{[17-19]}$. Estudos bem sucedidos de organomodificação foram realizados com argilas nacionais e os sais Dodigen $^{\circledR}$ (cloreto de alquila dimetil benzil amônio - Figura 3a), Praepagen $^{\circledR}$ (cloreto de estearil dimetil amônio - Figura 3b), Genamin $^{\circledR}$ (cloreto de cetil trimetil amônio - Figura 3c) e Cetremide ${ }^{\circledR}$ (brometo de cetil trimetil amônio - Figura 3d). Por difração de raios $\mathrm{X}$, notou-se que houve a intercalação dos sais quaternários de amônio entre as camadas de silicato, através do deslocamento do pico para ângulos menores e do aumento na distância interlamelar basal de valores entre 12 a $16 \AA$ para valores situados entre 20 e $40 \AA$, sendo estes valores dependentes do comprimento da molécula orgânica, do número dos grupos alquila ligados ao nitrogênio destes sais e de seu grau de inclinação no plano do argilomineral ${ }^{[19-22]}$.

\section{Nanocompósitos Polímero/Argila Brasileiros}

Publicações brasileiras sobre nanocompósitos polímero/argila em revistas nacionais e estrangeiras começaram a despontar na literatura a partir de 2002 e desde então, um número expressivo de artigos vem sendo publicado nas mais diversas revistas especializadas. A seguir, são apresentados exemplos dos avanços<smiles>[R]C(C)CCc1ccccc1</smiles>

(a)

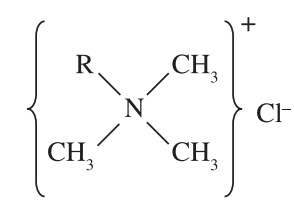

$\mathrm{R}=$ predominantemente $\mathrm{C}_{16}$

(c)

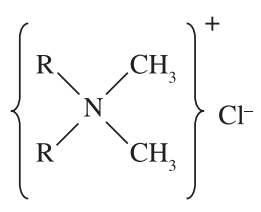

$\mathrm{R}=\mathrm{C}_{16} / \mathrm{C}_{18}$

(b)

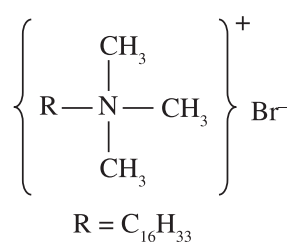

(d)
Figura 3. Estrutura molecular dos sais quaternários de amônio: a) Dodigen ${ }^{\circledR}$; b) Praepagen ${ }^{\circledR}$; c) Genamin ${ }^{\circledR}$; e d) Cetremide ${ }^{\circledast[22]}$. científicos referentes aos estudos brasileiros, estando estes divididos em polímeros de uso geral, polímeros de engenharia e outros tipos de polímeros.

\section{Nanocompósitos de Polímeros de Uso Geral}

Os polímeros de uso geral são caracterizados por serem utilizados em aplicações de baixo custo devido à facilidade de processamento e baixo nível de exigência mecânica. Uma forma de agregar valor a estas commodities é a formação de nanocompósitos, de forma a melhorar suas propriedades. Um grande número de trabalhos enfoca o uso de polietileno (PE) na produção de nanocompósitos e a relação de suas propriedades com o tipo e conteúdo de argila empregados $^{[22-29]}$.

O grupo de pesquisa em nanocompósitos da UFCG estudou o preparo de nanocompósitos de PE/argila brasileira bentonita organomodificada com diferentes sais quaternários de amônio por intercalação por fusão. Para um mesmo conteúdo de argila, neste caso $3 \%$ em massa, a morfologia dos nanocompósitos preparados com diferentes argilas organofílicas foi influenciada, como se pôde observar a partir dos difratogramas de raios $\mathrm{X}$ e das micrografias obtidas por microscopia eletrônica de transmissão.

$\mathrm{O}$ pico relativo à distância basal do nanocompósito PE/MMT, 13,3 Å, era próximo da posição do pico obtido para a argila não modificada, cuja distância basal era de $12,5 \AA$ A. Sendo assim, este discreto aumento seria relativo a uma pequena quantidade de cadeias de PE intercaladas entre as lamelas de argila. O aumento mais considerável da distância para $30 \AA$ e o aparecimento de um ombro correspondente à distância de 15,6 ̊̊ referente ao nanocompósito com argila organomodificada com Cetremide ${ }^{\circledR}$ sugeria a intercalação de um maior número de moléculas de PE entre as camadas de argila, sendo que o nanocompósito com argila organomodificada com Genamin $^{\circledR}$ apresentou o mesmo tipo de comportamento. Além disso, o nanocompósito preparado com argila organomodificada com Praepagen ${ }^{\circledR}$ apresentou, além dos elementos descritos nos últimos nanocompósitos, um terceiro ombro em $12,5 \AA$ A. E ainda, pelo fato de o nanocompósito com argila organomodificada com Dodigen ${ }^{\circledR}$ apresentar distância basal na mesma posição da argila organofílica, entender-se-ia que não houve intercalação do polímero, mas, como houve alargamento do pico, suspeitava-se que houvesse ocorrido intercalação parcial.

A partir das micrografias de cada um destes nanocompósitos, corroboraram-se as explicações relacionadas aos difratogramas de raios $\mathrm{X}$, ou seja, todos os nanocompósitos apresentavam camadas intercaladas distribuídas na matriz polimérica com grandes aglomerados e bem espaçadas, a exceção do nanocompósito com argila organomodificada com Praepagen que exibia uma morfologia intercalada com predominância de partículas de argila menores e dispersas na matriz polimérica. Tal diferença no comportamento foi explicada com base na diferença de estrutura de sal utilizado para modificar as argilas, visto que, o Praepagen ${ }^{\circledR}$ (Figura 3b), possui comprimento de cadeia maior que os outros sais, além de dois grupos alquila ligados ao átomo de nitrogênio. Assim, a disposição das cadeias orgânicas no sal teria proporcionado uma maior interação do polímero com as lamelas de argila.

Com relação ao comportamento térmico destes nanocompósitos, embora a presença de argila organofílica no nanocompósito possa catalisar a degradação da matriz polimérica, neste caso, a estabilidade térmica foi melhorada, visto que os picos das curvas de termogravimetria derivada com relação à temperatura máxima de decomposição eram aproximadamente 515, 520, 554, 527, 527 e $526{ }^{\circ} \mathrm{C}$ para o PE puro, nanocompósito preparado com argila não modificada e nanocompósitos preparados com argila organomodificada com sal Genamin ${ }^{\circledR}$, Cetremide $^{\circledR}$, Dodigen ${ }^{\circledR}$ 
e Praepagen ${ }^{\circledR}$, respectivamente. A maior estabilidade térmica foi originada pelo fato de as camadas de silicato agirem como retardantes ao aquecimento e transferência de massa durante a combustão ${ }^{[30-36]}$.

Além do efeito da organomodificação da argila, a influência do uso de PE graftizado com anidrido maleico (PE-g-MA) também foi investigada para formação de nanocompósitos por intercalação por fusão ${ }^{[37]}$. Por difração de raios X (Figura 4), notou-se que o sistema composto por PE/ 3\% em massa de argila organomodificada com Cetremide ${ }^{\circledR}$ (ACT) apresentou um deslocamento do ângulo $2 \theta=2,38^{\circ}$ e $\mathrm{d}_{001}=37,0 \AA$ em relação ao difratograma da argila organomodificada, correspondendo à intercalação das moléculas de PE entre as camadas da argila; e um segundo ombro a $2 \theta=4,6^{\circ}$ $(19,0 \AA)$ seria um indício de que uma pequena quantidade das lamelas da argila organomodificada não foi intercalada pelas moléculas de PE uma vez que tais valores eram muito próximos do pico característico da argila em questão. O sistema PE-g-MA/ $3 \%$ em massa de ACT não apresentou picos no difratograma, o que indicaria a presença de morfologia esfoliada e o híbrido PE/PE-gMA/ 3\% em massa de ACT apresentou um aumento da distância basal para $42,3 \AA$ A, relativo à intercalação de moléculas do PE entre as lamelas de argila e a presença de um segundo ombro a $2 \theta=4,72^{\circ}$ $(19,2 \AA)$, indicando novamente que uma pequena porção das camadas de argila não foi intercalada pelas moléculas de PE.

Tais resultados foram confirmados por microscopia eletrônica de transmissão. A micrografia referente ao nanocompósito preparado somente com PE (Figura 5a) apresentava aglomerados grandes e pequenos cujas camadas de argila na matriz de PE se caracterizavam por uma morfologia intercalada, conforme os resultados obtidos por

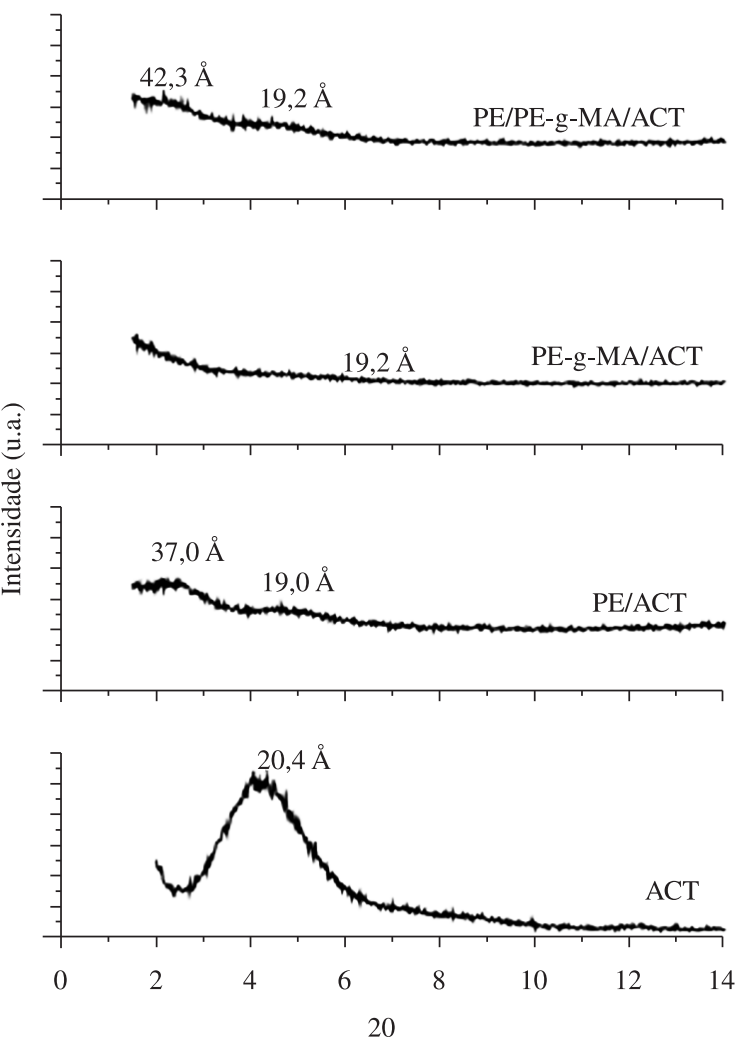

Figura 4. Difratogramas da argila organomodificada com Cetremide (ACT) e dos sistemas PE/ 3\% em massa de ACT, PE-g-MA/ 3\% em massa de ACT e PE/ PE-g-MA/ 3\% em massa de $\mathrm{ACT}^{[38]}$. difração de raios X (DRX). A micrografia do sistema preparado com PE-g-MA (Figura 5b) também denotou um grande aglomerado, contudo este continha várias partículas de argila muito pequenas com comprimentos em torno de $100 \mathrm{~nm}$ (destacadas por setas na figura) evidenciando esfoliação parcial e confirmando, portanto, o desaparecimento do pico basal no difratograma da Figura 4. Na micrografia do híbrido com PE e PE-g-MA (Figura 5c), várias são as partículas de argila dispersas no PE de comprimentos na ordem de $500 \mathrm{~nm}$, comprovando o resultado obtido por DRX que indicava a intercalação das moléculas de PE entre as lamelas da argila. Uma vez que a natureza apolar do PE prejudica a interação com as camadas de argila, entendeu-se que o uso de PE-g-MA melhorou as interações entre PE e camadas de argila, refletindo inclusive em uma melhoria da resistência ao impacto e em uma menor inflamabilidade, denotada pela redução da velocidade de queima, dos nanocompósitos de PE com PE-g-MA ${ }^{[38]}$.

O polipropileno (PP) é um polímero bastante utilizado graças ao seu baixo custo e grande versatilidade de aplicações, possuindo também um número relevante de estudos sobre a formação de nanocompósitos ${ }^{[39-49]}$. O grupo de Tavares utilizou técnicas de RMN para determinar a formação de nanocompósito e compreender a morfologia resultante ${ }^{[50]}$. Em um estudo, duas técnicas de intercalação por fusão - mistura de PP com argila montmorilonita brasileira por reômetro Haake e por extrusão - foram investigadas. Espectros de $\mathrm{RMN}{ }^{13} \mathrm{C}$ das amostras com argila revelaram modificações referentes ao empacotamento e ordenação das cadeias em relação à resina PP pura e difratogramas de raios $\mathrm{X}$ detectaram que somente a extrusão foi efetiva na formação de nanocompósitos ${ }^{[51,52]}$.

Nanocompósitos de polímeros estirênicos ${ }^{[53]}$ também foram extensamente estudados pelo grupo de Demarquette, especialmente no tocante às propriedades reológicas ${ }^{[54-57]}$. Em um destes trabalhos, estudou-se a influência do conteúdo de argila na resistência à luz ultravioleta de nanocompósitos preparados com PS e argila organofílica por injeção. Foi observado que, após 12 semanas de exposição à UV, a massa molecular média do PS puro foi reduzida a $85 \%$, enquanto que, nos nanocompósitos preparados com 2,5, 5,0 e 7,5\% em massa de PS, a redução foi de 83,5, 79,5 e 72,6\%, respectivamente, ou seja, a maior resistência com o aumento do conteúdo de argila é devida à função de proteção das camadas de argila contra luz UV e do efeito de barreira contra a difusão do oxigênio $^{[58]}$.

A utilização de diferentes métodos de intercalação por fusão como também a influência da granulação do composto obtido na forma de pó foram estudadas por Rodolfo Jr. e Mei no preparo de nanocompósitos de poli(cloreto de vinila) (PVC). Concluiuse que o uso de PVC granulado ou em pó não influenciava a morfologia final do nanocompósito, neste caso, híbrida intercalada/ parcialmente esfoliada, e a resistência térmica da amostra era somente influenciada pela presença do sal quaternário de amônio, que era menor em relação à resina $\mathrm{PVC}^{[59]}$. Marini, Branciforti $\mathrm{e}$ Lotti estudaram nanocompósitos de copoli (etileno-acetato de vinila) (EVA) com argila natural Cloisite ${ }^{\circledR}$ sódica e Cloisite $^{\circledR} 30 \mathrm{~B}$ organomodificada. Neste trabalho, estudos reológicos realizados com nanocompósitos de diferentes tipos de copoli (etileno-acetato de vinila) com MMT natural e organomodificada demonstraram que quanto menor era a viscosidade do EVA, maior era a tendência de formarem-se nanocompósitos esfoliados enquanto que com maiores viscosidades do EVA, houve uma diminuição no espaço entre as lamelas de argila, muito provavelmente pela saída das moléculas de surfactante orgânico com altas tensões ${ }^{[60]}$.

Estudos de nanocompósitos de poliuretano (PU) desenvolvidos por grupos de pesquisas brasileiros também são encontrados na literatura $^{[61]}$. Por exemplo, nanocompósitos de PU/MMT foram 


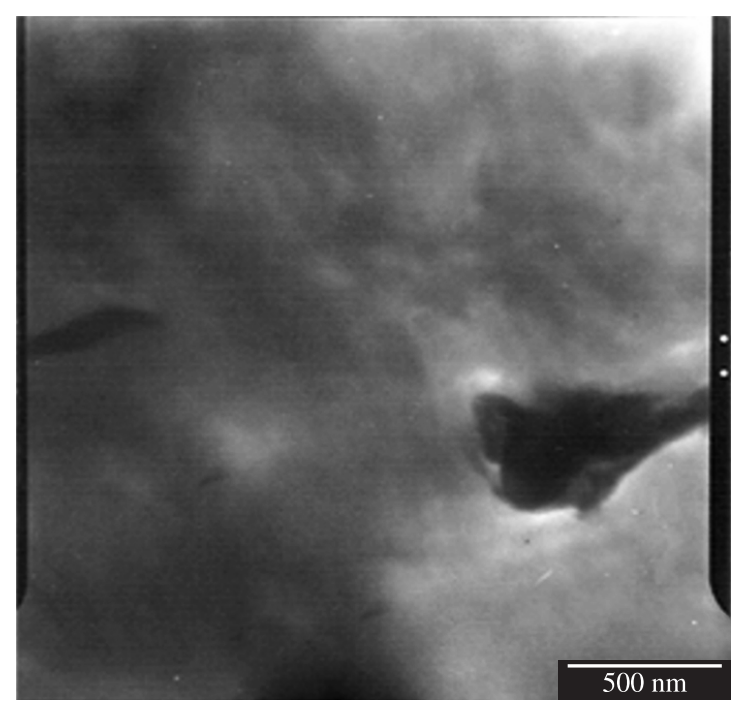

(a)

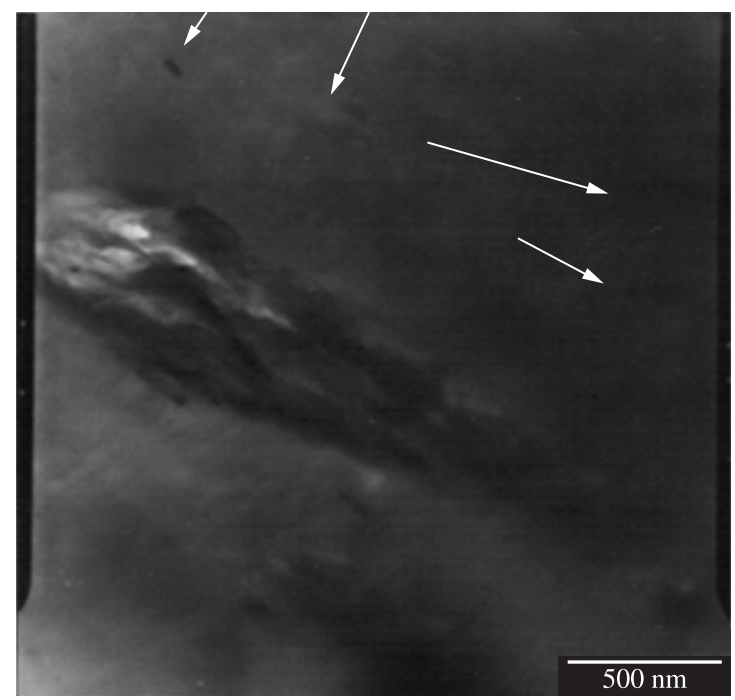

(b)

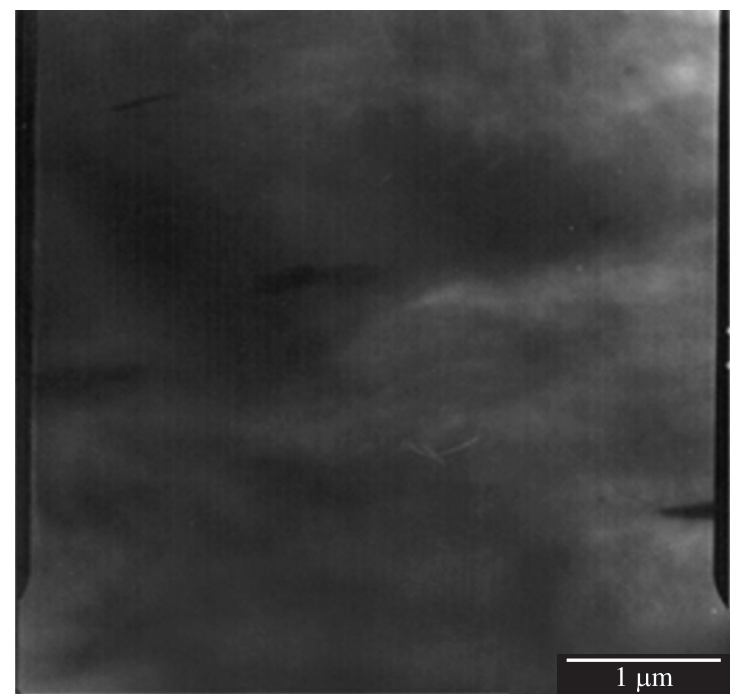

(c)

Figura 5. Micrografias dos nanocompósitos com 3\% em massa de argila organomodificada com Cetremide ${ }^{\circledR}$ (ACT): a) PE/ACT; b) PE-g-MA/ACT; e c) PE/ PE-g-MA/ACT ${ }^{[38]}$. preparados pelo grupo de Oréfice através da dispersão por solução em meio aquoso, apresentando, pelo acréscimo de $1 \%$ em massa de argila, aumento em $20 \%$ na tensão de ruptura em relação à resina original ${ }^{[62]}$. Contudo, o acréscimo de $5 \%$ em massa de argila resultou no decréscimo desta propriedade mecânica ${ }^{[63]}$. Aplicações biomédicas como sistemas de liberação de fármacos, nas quais as nanopartículas aumentaram a taxa de liberação de acetato de dexametasona graças às nanopartículas possuírem uma superfície hidrofílica que aumenta a absorção de água e acelera a hidrólise do polímero $^{[64]} \mathrm{e}$; membranas para regeneração periodontal, que forneceram um ambiente adequado para adesão e proliferação de cementoblastos $^{[65]}$, foram desenvolvidas pelos nanocompósitos preparados em meio aquoso.

Nanocompósitos de epóxi com fibras de vidro e argila MMT organomodificada foram preparados ${ }^{[6,67]}$, resultando em uma morfologia intercalada com aumento de até $10 \%$ na dureza e $9 \%$ na resistência à fratura ${ }^{[68]}$.

A formação de nanocompósitos de álcool polivinílico (PVA) com argila MMT natural foi investigada pelo grupo de Pessan, na ausência ${ }^{[69]}$ e na presença de micropartículas de poliéster (PES). Na presença de PES, a afinidade química entre os três componentes do sistema teve um papel importante na dispersão da argila e na cristalinidade do PVA. Na ausência de PES, observou-se uma maior dispersão entre PVA e MMT pelo fato de o MMT induzir a nucleação do PVA, enquanto que nos sistemas com PES, a adição de argila favoreceu o crescimento dos cristalitos de PVA, o aumento do grau de cristalinidade e da temperatura de fusão ${ }^{[70,71]}$.

\section{Nanocompósitos de Polímeros de Engenharia}

Os polímeros de engenharia apresentam propriedades mecânicas e químicas superiores aos polímeros de uso geral. Alguns trabalhos brasileiros sobre a produção de nanocompósitos com polímeros de engenharia são encontrados na literatura. Com maior número de publicação, têm-se os nanocompósitos de poliamida (PA) ${ }^{[22,72-79]}$. Pita e seus colaboradores, além de estudarem o efeito da organomodificação de MMT argentina com $\varepsilon$-caprolactama e com sal quaternário de amônio em nanocompósitos de PA $6^{[80]}$, também estudaram a formação de nanocompósitos em blendas de PA 6 e PE com estas mesmas argilas por intercalação por fusão, através de um reômetro de torque. A formação de blendas de PA 6 e PE foi feita para superarem-se as desvantagens provenientes de cada polímero; o PE possui fraca interação com superfícies minerais, dificultando a formação de nanocompósitos por intercalação por fusão e a PA 6 é frágil, com baixa estabilidade dimensional, mas, com habilidade para formação de nanocompósitos. Por microscopia eletrônica de transmissão, observou-se a existência de separação de fases, mas também, de camadas de argila bastante dispersas na matriz polimérica, formando uma morfologia parcialmente intercalada/ esfoliada ${ }^{[81]}$.

Outro polímero bastante estudado para o preparo de nanocompósitos é o poli(tereftalato de etileno) (PET) ${ }^{[82-88]}$, polímero largamente empregado na indústria de embalagens. Dentre os trabalhos publicados por Pessan e colaboradores ${ }^{[89-91]}$, pode-se citar uma pesquisa na qual se estuda a influência do tipo de argila (as organofílicas Viscogel, Inpaltone e montmorilonita sódica organomodificada com cloreto de dialquildimetilamônio) no preparo de nanocompósitos de PET por intercalação por fusão, através do uso de um reômetro de torque. As argilas montmorilonita organomodificada e Viscogel não foram úteis na formação de nanocompósito por serem altamente empacotadas, impedindo a entrada das cadeias poliméricas e demonstrando que não bastava existir compatibilidade entre argila e polímero e sim, a existência de espaço livre para inserção das cadeias. Em contrapartida, a 
argila Inpaltone, por possuir menores densidades de população de cátions orgânicos entre as camadas de argila e de empacotamento interlamelar, possibilitou a formação de um nanocompósito quase totalmente esfoliado ${ }^{[92]}$.

Outro poliéster, o poli (tereftalato de trimetileno) (PTT), foi objeto de estudo para o preparo de nanocompósito com argila MMT organomodificada por reômetro de torque, um método de intercalação por fusão. Neste estudo, notou-se que o acréscimo do compatibilizante E-AE-GMA, um terpolímero randômico de $68 \%$ em massa de etileno, $24 \%$ de éster acrílico e $8 \%$ de metacrilato de glicidila, melhorou as interações entre o PTT e a argila, promovendo a formação de nanocompósitos híbridos intercalados, com apenas um passo de mistura ou esfoliados com dois passos de mistura, enquanto que sua ausência resultou em nanocompósitos intercalados independentemente do número de passos de mistura ${ }^{[93]}$.

$\mathrm{O}$ efeito de vários solventes foi estudado no processo de dispersão por solução para a formação de nanocompósitos acetato de celulose (CA) com argila MMT. Observou-se que a influência do solvente é decisiva em dois aspectos, na dispersão da argila e na solubilidade do polímero. Com interações favoráveis entre a argila e o solvente, principalmente nos casos em que o solvente foi misturado com água, ocorreu maior grau de dispersão e delaminação da argila na presença das cadeias de CA, graças a específicas interações polares, ligações de hidrogênio e formação de complexo entre as cadeias poliméricas e as moléculas de solvente, sendo, portanto, a escolha do solvente um fator muito importante para formação da morfologia desejada ${ }^{[94]}$.

Com polissulfona (PSf), foram preparadas por Anadão et al. membranas nanocompósitas de PSf e argila montmorilonita sódica de Wyoming. Um aumento de 29 e $116{ }^{\circ} \mathrm{C}$ nas temperaturas inicial e final de degradação foi observado para o sistema preparado com $3 \%$ em massa de MMT em comparação com a PSf pura graças à propriedades de barreira que impedem a difusão de moléculas de oxigênio no material, além do efeito labirinto destas camadas dispersas que atrasam a volatilização. Ambos alongamento na ruptura e resistência mecânica dos nanocompósitos apresentaram discreta melhoria em relação à PSf pura pelas interações existentes entre cadeias poliméricas e lamelas de argila, bem como, ao alinhamento das camadas de argila na direção da deformação, permitindo maiores deformações. Além disso, o potencial das membranas nanocompósitas para aplicações em filtração de soluções aquosas e em estações de tratamento de água foi denotado através da diminuição da energia livre de hidratação com o acréscimo de argila, parâmetro intrinsecamente ligado à hidrofilicidade da superfície do material ${ }^{[95-97]}$.

\section{Nanocompósitos de Outros Tipos de Polímeros}

A condutividade de um polímero condutor, comparável a de semicondutores, é dependente do ordenamento das cadeias poliméricas que pode ser atingido através da formação de nanocompósitos. Um dos polímeros condutores mais estudados nesta área é a polianilina (PANI).

A PANI pertence à classe dos polímeros condutores e tem como desvantagem sua pobre estabilidade ambiental e baixas resistências mecânica e às intempéries, tornando conveniente o preparo de nanocompósitos, pois quando o polímero está confinado em ambiente de dimensões nanométricas, suas propriedades são melhoradas ${ }^{[98]}$. Nanocompósitos de PANI com argila MMT na forma sódica por dispersão em solução após polimerização ex situ e por polimerização in situ foram preparadas pelo grupo de Temperini ${ }^{[99]}$. Em ambos os casos, a morfologia era do tipo intercalada. Por Raman e ressonância paramagnética eletrônica (EPR), notou-se que os polímeros possuíam estruturas diferentes, o que acabou por refletir em diferentes medidas de condutividade, sendo esta propriedade maior para o nanocompósito produzido por dispersão em solução ${ }^{[100]}$. Resultados de espectroscopia fotoelétrica de raios X (XPS) sugeriram que a estrutura polimérica intercalada produzida por polimerização in situ pudesse estar adsorvida na superfície externa e/ ou na aresta do cristal $^{[101,102]}$. Por microscopia eletrônica de varredura (MEV), notou-se que, com a variação da quantidade de argila, a morfologia do nanocompósito é alterada. A condutividade dos nanocompósitos variavam de $10^{-1}$ a $10^{-4}$ S.cm ${ }^{-1}$, conforme a proporção mássica de polímero/argila diminuía ${ }^{[103]}$.

O poli (óxido de etileno) (PEO) é um polímero eletrolítico com alta condutividade, de caráter cristalino, o que inibe a mobilidade catiônica. Para redução da cristalinidade deste polímero e conseqüente melhoria da condutividade do material resultante, podem-se incorporar as cadeias poliméricas em nanofases inorgânicas $^{[104]}$. Sabe-se ainda que o acréscimo de plastificante $\gamma$-butirolactona em sistemas com um polímero derivado de PEO, o poli (óxido de etileno-co-epicloridrina), resulta em um aumento da condutividade, mas, também, prejudica as propriedades mecânicas do sistema, não compensando o ganho na condutividade. Neste sistema, a tecnologia de nanocompósitos polímero-argila, através da dispersão por solução, foi benéfica para o aumento da condutividade iônica de 0,16 a 0,28 e a $0,34 \mathrm{mS} . \mathrm{cm}^{-1} \mathrm{com}$ a adição de 1 e $3 \%$ em massa de MMT, respectivamente, atingindo um platô em $7 \%$ em massa de MMT e para o aumento da resistência mecânica promovido pela solidificação do eletrólito. Ainda, quando este nanocompósito foi aplicado em célula solar sensibilizada por corante, notou-se que sua eficiência apresentava valores bem próximos em células preparadas com eletrólitos sólidos, demonstrando ser bastante promissora para esta aplicação ${ }^{[105]}$.

A formação de nanocompósitos de polibenzidina (PBZ), outro polímero condutor, com argila catiônica MMT através de polimerização in situ foi estudada por Do Nascimento, Constantino e Temperini. Por MEV e DRX, comprovou-se que a orientação das cadeias poliméricas era paralela em relação às camadas de argila, sendo um nanocompósito do tipo intercalado. A condutividade da PBZ intercalada era da mesma ordem de magnitude da resina PBZ, sugerindo que a intercalação do polímero poderia aumentar a condução eletrônica devido à melhora no arranjo supramolecular das cadeias poliméricas ${ }^{[106]}$.

No tocante aos polímeros biodegradáveis, pode-se mencionar o polihidroxibutirato (PHB) na produção de nanocompósitos. Tavares et al. estudaram a influência de diferentes solventes (clorofórmio, dimetilcloreto e tetrahidrofurano) no processo de dispersão por solução na cristalinidade do PHB e nas suas interações com a argila organomodificada Viscogel através da técnica de ressonância magnética nuclear de baixo campo, freqüentemente utilizada pelo grupo. Uma vez que a relaxação spinlattice do hidrogênio é controlada pelo processo de interação dipolar intermolecular, o maior tempo de relaxamento apresentado pelo sistema que continha dimetilcloreto denotava a boa solubilização que ocorreu entre os componentes do nanocompósito, demonstrando esta ser uma boa ferramenta para compreensão da morfologia do nanocompósito ${ }^{[107]}$.

Com o objetivo de produzirem-se dispositivos de liberação de fármacos, o polietilenoglicol $(\mathrm{PEG})^{[108]}$, a polivinilpirrolidona $(\mathrm{PVP})^{[109]}$ e a quitosana ${ }^{[110]}$ foram empregados para a formação de nanocompósitos intercalados com argila organomodificada, a Viscogel B8, por dispersão por solução. Todos apresentaram bom desempenho como excipientes potenciais matrizes controladoras da cinética de liberação de fármacos, de acordo com ensaio de liberação in vitro efetuado para o fármaco dapsona em suco gástrico simulado. 
Ainda com relação a aplicações biomédicas, a argila MMT natural e dois tipos de argila MMT organofílica $\left(\right.$ Cloisite $^{\circledR} 20 \mathrm{~A}$ e 30B) foram utilizados no preparo de nanocompósitos intercalados/ esfoliados para resinas dentárias por polimerização in situ objetivando a melhoria na resistência ao atrito, baixo encolhimento polimérico, dentre outras propriedades importantes para emprego em implantes dentários ${ }^{[111]}$.

Polímeros naturais como os polissacarídeos também foram estudados para o preparo de nanocompósitos. Com a adição de plastificantes apropriados, produziram-se nanocompósitos de amido intercalados, cuja morfologia e espaçamento basal eram dependentes da estrutura química do polímero e do plastificante ${ }^{[112]}$.

Alguns trabalhos também apresentam o uso do látex, seja ele natural ${ }^{[13,114]}$ ou sintético ${ }^{[115]}$, no preparo de nanocompósitos com argila. Como exemplo, a argila MMT sódica e argilas derivadas com outros íons entre suas galerias (potássio, lítio e cálcio) foram empregadas na produção de nanocompósitos de látex de estirenoacrílico por dispersão em solução, utilizando como solvente a água, com o intuito de compreender-se a influência dos contra-íons na morfologia e nas propriedades dos nanocompósitos resultantes. Para os íons monovalentes, observou-se um aumento de $200 \%$ de resistência à tração, se comparado com o polímero puro, resultado da morfologia intercalada/ esfoliada resultante. No caso do íon cálcio, pela menor extensão da esfoliação, as propriedades mecânicas de seu nanocompósito eram um pouco inferiores. Neste estudo, entendeuse ainda que os contra-íons possuíam um papel fundamental na formação de nanocompósitos, pois com o uso de diferentes íons, novos materiais podem ser formados graças à formação de ligações iônicas que promovem a adesão entre polímero e argila, ambos detentores de carga negativa ${ }^{[115]}$.

\section{Considerações Finais}

Grande parte dos trabalhos nacionais encontra-se focada na produção de nanocompósitos de termoplásticos, havendo um número expressivo também na formação de nanocompósitos com polímeros condutores. No caso dos nanocompósitos termoplásticos, a intercalação por fusão foi largamente empregada pelos pesquisadores brasileiros, em segundo lugar, com um número menor de trabalhos, a polimerização in situ foi uma técnica menos utilizada, enquanto que a síntese de nanocompósitos por dispersão por solução raramente foi objeto de pesquisa brasileira. Com relação às argilas utilizadas, pode-se afirmar que a grande maioria dos trabalhos fez uso de argilas organofílicas ou então, modificaram argilas naturais, em sua grande maioria importadas, a fim de tornálas organofílicas objetivando promover melhor interação entre a matriz polimérica e as lamelas de argila.

Ainda, com base nos registros da literatura desde os primórdios dos estudos de nanocompósitos até o início de 2010, nota-se que o maior número de artigos publicados foi originado por pesquisadores do estado de São Paulo. Um número bastante expressivo de artigos também foi publicado no estado da Paraíba por pesquisadores da UFCG, especialmente focados em nanocompósitos de PE e PA, diferentemente da pesquisa no estado de São Paulo, que permeia todos os tipos de polímeros e está distribuída em diferentes universidades do estado. Pesquisa bastante expressiva foi efetuada no estado do Rio de Janeiro, por pesquisadores do Instituto de Macromoléculas Professora Eloisa Mano da Universidade Federal do Rio de Janeiro. Importantes contribuições ao estudo de nanocompósitos também foram realizadas nos estados de Minas Gerais, Rio Grande do Sul, Santa Catarina e Paraná. Considerando que o Brasil é composto por 26 estados federados e um distrito federal, e que, seja de conhecimento dos autores, somente 7 estados contribuíram nas pesquisas direcionadas para nanocompósitos, seria interessante que grupos de pesquisas poliméricas de outros estados se envolvessem nesta área que demonstra ser bastante promissora sob os aspectos anteriormente mencionados.

Além disso, um número extremamente pequeno de artigos apresenta colaboração com indústrias do segmento de plásticos, bem como, com instituições de pesquisa estrangeiras, restringindose somente à participação nestas poucas pesquisas dos países Espanha, Itália, Estados Unidos, França e Argentina.

Para que a pesquisa brasileira em nanocompósitos evolua, entendemos que alguns aspectos devem ser considerados:

- a exemplo do grupo de pesquisa da UFCG que estuda as argilas da Paraíba, outros grupos de pesquisa devem realizar um estudo mais aprofundado do potencial de formação de nanocompósitos com argilas nacionais de forma a obter uma redução no custo do produto final, visto que, além das reservas de bentonita no estado da Paraíba, existem também reservas em outros estados brasileiros;

- é necessário um estudo comparativo com diversos e novos tipos de organomodificação das argilas, como o uso de surfactantes não-iônicos, de modo a produzirem-se nanocompósitos inteiramente esfoliados e possibilitar uma melhoria acentuada das propriedades de barreira, térmicas e mecânicas;

- uma vez que a formação de nanocompósitos de caráter completamente esfoliado ainda constitui um desafio para os pesquisadores brasileiros, pois, as pesquisas realizadas demonstram haver, além da esfoliação, certa porcentagem de intercalação e de aglomeração das partículas de argila, seria recomendável a intensificação de pesquisas acerca dos processos de esfoliação, de modo a minimizar a formação de nanocompósitos inomogêneos;

- o emprego de nanocompósitos no exterior vem gradativamente avançando nos diversos ramos da indústria. Para que o Brasil se torne independente e, ao mesmo tempo, competitivo em relação aos produtos que já fazem uso desta tecnologia, é necessário que as aplicações de nanocompósitos polímero/argila evoluam significativamente a partir de desenvolvimentos em setores onde poderiam estar presentes de forma mais contundente, a exemplo das aplicações na indústria automobilística. Parecenos serem também bastante promissoras as pesquisas sobre nanocompósitos de polímeros condutores, os quais espera-se que possam substituir, por exemplo, diodos emissores de luz (LED), cujo mercado é exponencial.

Por fim, deve-se ressaltar que a grande maioria dos trabalhos sobre nanocompósitos polímero/ argila no Brasil tem tido o apoio de agências financiadoras brasileiras de pesquisa, apoio este que tem sido decisivo para o forte desenvolvimento da área no âmbito acadêmico. Com esta colaboração e com uma perspectiva crescente de envolvimento industrial nas pesquisas e desenvolvimentos de produtos nanocompósitos, as tendências para o setor são de um incremento substancial da produção nas próximas décadas.

\section{Agradecimentos}

À FAPESP pela bolsa de doutorado concedida à Priscila Anadão.

\section{Referências Bibliográficas}

1. Alexandre, M. \& Dubois, P. - Mat. Sci. Eng. R, 28, p.1 (2000). http:// dx.doi.org/10.1016/S0927-796X(00)00012-7

2. Brigatti, M. F.; Galan, E. \& Theng, B. K. G. - "Structures and mineralogy of clay minerals", in: Handbook of Clay Science, cap. 2, Bergaya, F.; Theng, B. K. G. \& Lagaly, G. (eds.), Elsevier, Amsterdam, (2006). http://dx.doi.org/10.1016/S1572-4352(05)01002-0

3. Ke, Y. C. \& Stroeve, P. - "Polymer-Layered Silicate and Silica Nanocomposites", Elsevier B. V., Amsterdam (2005). 
4. Mittal, V. - Mater., 2, p.992 (2009). http://dx.doi.org/10.3390/ ma2030992

5. Choudalakis, G.; Gotsis, A. D. - Eur. Polym. J., 45, p.967 (2009). http:// dx.doi.org/10.1016/j.eurpolymj.2009.01.027

6. Yeh, J. -M.; Chang, K. -C. - J. Ind. Eng. Chem., 14, p.275 (2008). http://dx.doi.org/10.1016/j.jiec.2008.01.011

7. Pavlidou, S.; Papaspyrides, C. D. - Prog. Polym. Sci., 33, p.1119 (2008). http://dx.doi.org/10.1016/j.progpolymsci.2008.07.008

8. Ray, S. S.; Okamoto, M. - Prog. Polym. Sci., 28, p.1539 (2003).

9. Schmidt, D.; Shah, D.; Giannelis, E. P. - Curr. Opin. Solid. St. M., 6, p.205 (2002).

10. Rabello, M. S. - "Aditivação de Polímeros", Artliber editora, São Paulo (2000).

11. Carter, L. W.; Hendricks, J. G.; Bolley, D. S. - "Elastomer Reinforced With A Modified Clay", US Patent n. 2531396 (1950).

12. Okada, A.; Fukushina, Y.; Kawasumi, M.; Inagaki, S.; Usuki, A.; Sugiyama, S.; Kurauchi, S. \& Kamigaito, T. - "Composite material and process for manufacturing same", US Patent n. 4739007 (1988).

13. Stewart, R. - Plast. Eng., 5, p.22 (2004).

14. Radford, J. - "Enhanced barrier properties in containers and films", in: Annals of the Future of Nanomaterials Conference, Birminghan, jun (2004).

15. Souza, M. A.; Pessan, L. A. \& Rodolfo Junior, A. - Polímeros, 16, p.257 (2006). http://dx.doi.org/10.1590/S0104-14282006000400003

16. Kaneko, M. L. Q. A.; Torriani, I. \& Yoshida, I. V. P. - J Braz. Chem. Soc., 18, p.765 (2007). http://dx.doi.org/10.1590/S010350532007000400015

17. De Paiva, L.; Morales, A. \& Valenzuela-Díaz, F. R. - Appl. Clay Sci., 42, p.8 (2008). http://dx.doi.org/10.1016/j.clay.2008.02.006

18. Valenzuela-Díaz, F. R. - "Preparação, a nível de laboratório, de algumas argilas esmectíticas organofílicas", Tese de Doutorado, Universidade de São Paulo, Brasil (1994).

19. Leite, I. F.; Soares, A. P. S.; Carvalho, L. H.; Raposo, C. M. O.; Malta, O. M. L. \& Silva, S. M. L. - J. Therm. Anal. Calorim., 100, p.563 (2010). http://dx.doi.org/10.1007/s10973-009-0265-3

20. Leite, I. F.; Raposo, C. M. O. \& Silva, S. M. L. - Cerâmica, 54, p.303 (2008).

21. Silva, S. M. L.; Araújo, P. E. R.; Ferreira, K. M.; Canedo, E. L.; Carvalho, L. H. \& Raposo, C. M. O. - Polym. Eng. Sci., 49, p.1696 (2009). http://dx.doi.org/10.1002/pen.21399

22. Barbosa, R.; Araújo, E. M.; Maia, L. F.; Pereira, O. D.; Mélo, T. J. A. \& Ito, E. N. - Polímeros, 16, p.246 (2006).

23. Rodrigues, T.; Tavares, M. I. B.; Preto, M.; Soares, I. L. \& Moreira, A. - Int. J. Polym. Mater., 57, p.1119 (2008). http://dx.doi. org/10.1080/00914030802428716

24. Lotti, C.; Isaac, C. S.; Branciforti, M. C.; Alves, R. M.; Libermann, S. \& Bretas, R. E. S. - Eur. Polym. J., 44, p.1346 (2008). http://dx.doi. org/10.1016/j.eurpolymj.2008.02.014

25. Pettarin, V.; Fasce, L.; Pita, V. J. R.; Dias, M. L. \& Frontini, P. M. - Compos. Interf., 16, p.201 (2009). http://dx.doi. org/10.1163/156855409X402885

26. Pettarin, V.; Frontini, P. M.; Pita, V. J. R.; Dias, M. L. \& ValenzuelaDíaz, F. R. - Compos. Part A. -Appl S., 39, p.1822 (2008).

27. Pettarin, V.; Pita, V. J. R.; Fasce, L.; Seltzer, R.; Dias, M. L. \& Frontini, P. - Key Eng. Mat., 312, p.205 (2006). http://dx.doi.org/10.4028/www. scientific.net/KEM.312.205

28. Araújo, E. M.; Mélo, T. J. A.; Oliveira, A. D.; Araújo, H. L. D.; Araújo, K. D. \& Barbosa, R. - Polímeros, 16, p.38 (2006).
29. Junges, F.; Beauvalet, M. S.; Leal, B. C.; Casagrande, A. C. A.; Mota, F. F.; Mauler, R. S. \& Casagrande Junior, O. L. - J. Braz. Chem. Soc., 20, p.472 (2009). http://dx.doi.org/10.1590/S0103-50532009000300010

30. Barbosa, R.; Araújo, E. M.; Mélo, T. J. A. \& Ito, E. N. - Mat. Lett., 61, p.2575 (2007). http://dx.doi.org/10.1016/j.matlet.2006.09.055

31. Araújo, E. M.; Barbosa, R.; Oliveira, A. D.; Morais, C. R. S; Mélo, T. A. J. \& Souza, A. G. - J. Therm. Anal. Calor., 87, p.811 (2007). http:// dx.doi.org/10.1007/s10973-006-7758-0

32. Barbosa, R.; Araújo, E. M.; Melo, T. J. A. \& Ito, E. N. - Polímeros, 17, p.104 (2007).

33. Araújo, E. M.; Barbosa, R.; Rodrigues, A. W. B.; Melo, T. J. A. \& Ito, E. N. - Mat. Sci. Eng. A, 445-446, p.141 (2007).

34. Barbosa, R.; Araújo, E. M.; Mélo, T. J. A.; Ito, E. N. \& Hage Junior, E. - J. Nanosci. Nanotechnol., 8, p.1 (2008).

35. Araújo, E. M.; Oliveira, A. D.; Barbosa, R. \& Mélo, T. J. A. - Mat. Sci. For., 530-531, p.709 (2006).

36. Araújo, E. M.; Barbosa, R.; Morais, C. R. S.; Soledade, L. E. B.; Souza, A. G. \& Vieira, M. Q. - J. Therm. Anal. Calorim., 90, p.841 (2007). http://dx.doi.org/10.1007/s10973-006-7504-7

37. Araújo, E. M.; Kojuch, L. R.; Barbosa, R. Nóbrega, K. C. \& Mélo, T. J. A. - Remap, 3, p.20 (2008).

38. Brito, G. F.; Oliveira, A. D.; Araújo, E. M., Mélo, T. J. A.; Barbosa, R. \& Ito, E. N. - Polímeros, 18, p.170, 2008. http://dx.doi.org/10.1590/ S0104-14282008000200015

39. Martins, C. G.; Larocca, N. M.; Paul, D. R. \& Pessan, L. A. - Polymer, 50, p.1743 (2009). http://dx.doi.org/10.1016/j.polymer.2009.01.059

40. Chinellato, A. C.; Vidotti, S. E.; Hu, G-H. \& Pessan, L. A. - J. Polym. Sci. Pol. Phys., 46, p.1811, 2008. http://dx.doi.org/10.1002/polb.21514

41. Paiva, L. B.; Morales, A. R. \& Guimarães, T. R. - Mat. Sci. Eng. A Struct., 447, p.261 (2007).

42. Rodrigues, A. W.; Brasileiro, M. I.; Araújo, W. D.; Araújo, E. M.; Neves, G. A. \& Mélo, T. J. A. - Polímeros, 17, p.219 (2007).

43. Silva, S. M. L.; López-Manchado, M. A. \& Arroyo, M. - J. Nanosci. Nanotechnol., 7, p.4456 (2007).

44. Ramos Filho, F. G.; Ávila Junior, M. M.; Santana, L. D. L.; Melo, T. J. A. \& Silva, S. M. L. - Matéria, 10, p.24 (2005).

45. Araújo, S. S.; Paiva, G. P.; Carvalho, L. H. \& Silva, S. M. L. - Matéria, 9, p.426 (2004).

46. Gianelli, W.; Ferrara, G. Camino, G.; Pellegatti, G.; Rosenthal, J. \& Trombini, R. C. - Polymer, 46, p.7037 (2005). http://dx.doi. org/10.1016/j.polymer.2005.05.149

47. Ramos Filho, F. G.; Mélo, T. J. A.; Rabello, M. S. \& Silva, S. M. L. - Polym. Degrad. Stab., 89, p.383 (2005). http://dx.doi.org/10.1016/j. polymdegradstab.2004.12.011

48. Paiva, L. B.; Morales, A. R. \& Guimarães, T. R. - Polímeros, 16, p.136 (2006).

49. Araújo, S. S.; Andrade, D. L. A. C. S.; Carvalho, L. H.; Gomes, A. S. \& Silva, S. M. L. - Plást. Ind., 88, p.104 (2005).

50. Tavares, M. I. B.; Nogueira, R. F.; Gil, R. A. S. S.; Preto, M.; Da Silva, E. O.; Silva, M. B. R. \& Miguez, E. - Polym. Test., 26, p.1100 (2007).

51. Tavares, M. I. B.; Nogueira, R. F. \& San Gil, R. A. S. - J. Metast. Nanocrys. Mater., 22, p.71 (2004).

52. Tavares, M. I. B.; Nogueira, R. F.; San Gil, R. A. S. \& Silva, N. M. Ann. Magn. Reson., 22, p.60 (2004).

53. Arioli, R.; Gonçalves, O. H.; Castellares, L. G.; Da Costa, J. M.; Araújo, P. H.; Machado, R. \& Bolzan, A. - Macromol. Symp., 245-246, p.337 (2006). http://dx.doi.org/10.1002/masy.200651346

54. Moraes, R. P.; Valera, T. S.; Demarquette, N. R; Oliveira, P. C. Silva, M. L. C. P. \& Santos, A. M. - J. Appl. Polym. Sci., 112, p.1949 (2009). http://dx.doi.org/10.1002/app.29309 
55. Carastan, D. J.; Demarquette, N. R.; Vermoget, A. \& Varlot, K. M. Rheol. Acta, 47, p.521 (2008). http://dx.doi.org/10.1007/s00397-0080283-2

56. Carastan, D. J. \& Demarquette, N. R. - Macromol. Symp., 233, p.152 (2006). http://dx.doi.org/10.1002/masy.200690012

57. Moraes, R. P.; Santos, A. M.; Oliveira, P. C.; Souza, F. C. T.; Do Amaral, M.; Valera, T. S. \& Demarquette, N. R. - Macromol. Symp., 245-246, p.106 (2006). http://dx.doi.org/10.1002/masy.200651314

58. Oliveira, C. F. P.; Carastan, D. J. ; Demarquette, N. R. \& Fechine, G. J. M. - Polym. Eng. Sci., 48, p.1511 (2008). http://dx.doi.org/10.1002/ pen. 21120

59. Rodolfo Jr., A. \& Mei, L. H. I. - Polímeros, 19, p.1, 2009. http://dx.doi. org/10.1590/S0104-14282009000100006

60. Marini, J.; Branciforti, M. C. \& Lotti, C. - Polym. Advan. Technol., 21, p.408 (2010).

61. Pizzatto, L.; Lizot, A; Fiorio, R.; Amorim, C. L.; Machado, G.; Giovanela, M.; Zattera, A. J. \& Crespo, J. S. - Mat. Sci. Eng. C - Bio S., 29, p.474 (2009).

62. Ayres, E. \& Oréfice, R. L. - Polímeros, 17, p.339 (2007). http://dx.doi. org/10.1590/S0104-14282007000400015

63. Ayres, E.; Oréfice, R. L. \& Sousa, D. - Macromol Symp., 245-246, p.330 (2006). http://dx.doi.org/10.1002/masy.200651345

64. Silva, G. R.; Ayres, E.; Oréfice, R. L. Moura, S. A. L.; Cara, D. C. \& Cunha Junior, A. S. - J. Drug Target., 17, p.374 (2009).

65. Pereira, I. M.; Carvalho, S.; Leite, M. F.; Pereira, M. M. \& Oréfice, R. L. - J. Appl. Polym. Sci., 14, p.254 (2009). http://dx.doi.org/10.1002/ app.30404

66. Ávila, A. F.; Donadon, L. V. \& Duarte, H. V. - Comp. Struct., 83, p. 324 (2008).

67. Ávila, A.; Duarte, H. V. \& Soares, M. I. - LAJSS, 3, p.3 (2006).

68. Ávila, A. F. \& Morais, D. T. S. - Comp. Struct., 87, p.55 (2009). http:// dx.doi.org/10.1016/j.compstruct.2007.12.009

69. Paranhos, C. M.; Soares, B. G.; Oliveira, R. N. \& Pessan, L. A. Macromol. Mater. Eng., 292, p.620 (2007). http://dx.doi.org/10.1002/ mame. 200700004

70. Paranhos, C. M.; Dahmouche, K.; Zaioncz, S.; Soares, B. G. \& Pessan, L. A. - J. Polym. Sci. Pol. Phys., 46, p.2618 (2008). http://dx.doi. org/10.1002/polb.21586

71. Paranhos, C. M.; Soares, B. G.; Machado, J. C.; Windmoller, D. \& Pessan, L. A. - Eur. Polym. J., 43, p.4882 (2007). http://dx.doi. org/10.1016/j.eurpolymj.2007.10.001

72. Souza, M. A.; Larocca, N. M.; Araújo, E. M. \& Pessan, L. A. Mater. Sci. Forum, 570, p.18 (2008). http://dx.doi.org/10.4028/www. scientific.net/MSF.570.18

73. Paz, R. A.; Leite, A. M. D.; Araújo, E. M.; Mélo, T. J. A.; Barbosa, R. \& Ito, E. N. - Polímeros, 18, p.341 (2008). http://dx.doi.org/10.1590/ S0104-14282008000400014

74. Araújo, E. M.; Araújo, K. D.; Paz, R. A.; Gouveia, T. R.; Barbosa, R. \& Ito, E. N. - J. Nanomat., 2009, p.1 (2009).

75. Medeiros, V. N; Araújo, E. M.; Maia, L. F.; Pereira, O. D.; Arimateia, R. R. \& Paz, R. A. - Polímeros, 18, p.302 (2008).

76. Araújo, E. M.; Mélo, T. J. A.; Santana, L. N. L.; Neves, G. A.; Ferreira, H. C.; Lira, H. L.; Carvalho, L. H.; Ávila Junior, M. M.; Pontes, M. K. G. \& Araújo, I. S. - Mat. Sci. Eng. B, 112, p.175 (2004).

77. Araújo, E. M.; Araújo, K. D. \& Gouveia, T. R. - Mat. Sci. Forum, 530-531, p.702 (2006).

78. Pankaj, A.; Araújo, W. D.; Oliveira, S. I.; Rodrigues, A. W.; Araújo, E. M. \& Mélo, T. J. A. - Remap, 1, p.45 (2006).

79. Paz, R. A.; Araújo, E. M.; Barbosa, R.; Pereira, O. D. \& Mélo, T. J. A. - Remap, 2, p.8 (2007).
80. Erdmann, E.; Dias, M. L.; Pita, V. J. R. R.; Monasterio, F. \& Destéfanis, H. A. - Mat. Sci. Forum, 570, p.78 (2008). http://dx.doi.org/10.4028/ www.scientific.net/MSF.570.78

81. Erdmann, E.; Dias, M. L.; Pita, V. J. R. R.; Destéfanis, H. A.; Monasterio, F. \& Acosta, D. - Macromol. Symp., 258, p.82 (2007). http://dx.doi.org/10.1002/masy.200751209

82. Calcagno C. I. W. ; Mariani C. M.; Teixeira S. R. \& Mauler, R. S. Compos. Sci. Technol., 68, p.2193 (2008). http://dx.doi.org/10.1016/j. compscitech.2008.03.012

83. Giraldi, A. L. F. M.; Bizarria, M. T. M.; Silva, A. A.; Velasco, J. I. D’Avila, M. A. \& Mei, L. H. I. - J. Appl. Polym. Sci., 108, p. 2252 (2008). http://dx.doi.org/10.1002/app.27280

84. Bizarria, M. T. M.; Giraldi, A. L. F. M.; De Carvalho, C. M; Velasco, J. I.; D’Avila, M. A. \& Mei, L. H. I. - J. Appl. Polym. Sci., 104, p.1839 (2007). http://dx.doi.org/10.1002/app.25836

85. Leite, I. F.; Raposo, C. M. O.; Carvalho, L. H. \& Silva, S. M. L. Matéria, 11, p.260 (2006).

86. Calcagno, C. I. W.; Mariani, C. M.; Teixeira, S. R. \& Mauler, R. S. - J. Appl. Polym. Sci., 111, p.29 (2009). http://dx.doi.org/10.1002/ app. 28977

87. Raposo, C. M. O.; Leite, I. F; Carvalho, L. H. \& Silva, S. M. L. Plástico Industrial, 106, p.106 (2007).

88. Calcagno, C. I. W.; Mariani, C. M.; Teixeira, S. R. \& Mauler, R. S. - Polymer, 48, p.966 (2007). http://dx.doi.org/10.1016/j. polymer.2006.12.044

89. Andrade, M. L. Q.; Manrich, S. \& Pessan, L. A. - J. Metastable Nanocryst. Mater., 22, p.51 (2004). http://dx.doi.org/10.4028/www. scientific.net/JMNM.22.51

90. Vidotti, S. E.; Chinellato, A. C.; Hu, G. -H. \& Pessan, L. A. - J. Polym. Sci. Pol. Phys., 45, p.3084 (2007). http://dx.doi.org/10.1002/ polb. 21311

91. Vidotti, S. E.; Chinellato, A. C.; Boesel, L. F. \& Pessan, L. A. J. Metastable Nanocryst. Mater., 22, p.57 (2004). http://dx.doi. org/10.4028/www.scientific.net/JMNM.22.57

92. Boesel, L. F. \& Pessan, L. A. - Mat. Sci. Forum, 403, p.89 (2002). http://dx.doi.org/10.4028/www.scientific.net/MSF.403.89

93. Fávaro, M.; Branciforte, M. C. \& Bretas, R. E. S. - Polym. Advan. Technol., 20, p.940 (2009).

94. Romero, R. B.; Leite, C. A. P. \& Gonçalves, M. C. - Polymer, 50, p.161 (2009). http://dx.doi.org/10.1016/j.polymer.2008.10.059

95. Anadão, P; Hespanhol, I.; Mierzwa, J. C.; Wiebeck, H. \& ValenzuelaDíaz, F. R. - "Processo de obtenção de membranas nanocompósitas de polisulfona com argila bentonita sódica para microfiltração e membranas nanocompósitas resultantes", BR Patent n. PI 0701474-0 (2007).

96. Anadão, P; Rabello, P. P.; Valenzuela-Díaz, F. R.; Wiebeck, H.; Hespanhol, I. \& Mierzwa, J. C. - Revista Aidis, 1 (2007).

97. Anadão, P.; Sato, L. F.; Wiebeck, H.; Valenzuela-Díaz, F. R. - Appl. Clay Sci., 48, p.127 (2010). http://dx.doi.org/10.1016/j.clay.2009.12.011

98. Soto-Oviedo, M.; Araújo, O. A.; Faez, R.; Rezende, M. \& De Paoli, M. A. - Synthetic Met., 156, p.1249 (2006). http://dx.doi.org/10.1016/j. synthmet.2006.09.003

99. Do Nascimento, G. M.; Constantino, V. R. L.; Landers, R. \& Temperini, M. L. A. - Polymer, 47, p.6131 (2006). http://dx.doi.org/10.1016/j. polymer.2006.06.036

100. Barbosa, P. S. M.; Honda, R. E.; Do Nascimento, G. M.; Izumi, C. M. S. \& Temperini, M. L. A. - "Caracterização espectroscópica dos nanocompósitos de polianilina na argila montmorillonita Syn1”, in: Anais da $26^{\circ}$ Reunião Anual da Sociedade Brasileira de Química, Águas de Lindóia - SP, mai (2003). 
101. Do Nascimento, G. M. - "Síntese e caracterização de nanocompósitos de polímeros condutores com argilas", Tese de Doutorado, Universidade de São Paulo, Brasil (2004).

102. Do Nascimento, G. M.; Constantino, V. R. L. \& Temperini, M. L. A. Macromol., 35, p.7535 (2002). http://dx.doi.org/10.1021/ma025571l

103. Do Nascimento, G. M.; Constantino, V. R. L.; Landers, R. \& Temperini, M. L. A. - Macromol., 37, p.9373 (2004). http://dx.doi.org/10.1021/ ma049054

104. Hikosaka, M. Y.; Pulcinelli, S. H.; Santilli, C. V.; Dahmouche, K. \& Craievich, A. F. - J. Non Crys. Sol., 352, p.3705 (2006). http://dx.doi org/10.1016/j.jnoncrysol.2006.02.124

105. Nogueira, A. F.; Ito, B. I.; Freitas, J. N. \& De Paoli, M. A. - J. Braz. Chem. Soc., 19, p.688 (2008). http://dx.doi.org/10.1590/S010350532008000400012

106. Do Nascimento, G. M.; Constantino, V. R. L. \& Temperini, M. L. A - J. Phys. Chem. B, 108, p.5564 (2004). http://dx.doi.org/10.1021/ jp037262i

107. Bruno, M.; Tavares, M. I. B.; Motta, L. M.; Miguez, E.; Preto, M. \& Fernandez, A. O. R. - Mat. Res., 11, p.483 (2008). http://dx.doi. org/10.1590/S1516-14392008000400018
108. Dornelas, C. B.; Resende, D. K.; Rocha, H. V. A.; Gomes, A. S.; Tavares, M. I. B.; Coutinho, S. S. S. \& Cabral, L. M. - Polímeros, 18, p.222 (2008). http://dx.doi.org/10.1590/S0104-14282008000300007

109. Dornelas, C. B.; Gomes, A. S.; Tavares, M. I. B. \& Resende, D. K. - Polímeros, 18, p.187 (2008). http://dx.doi.org/10.1590/S010414282008000200017

110. Rocha, H.; Gomes, A. S. \& Dornelas, C. B. - Polym. -Plast. Technol., 47, p.1256 (2008). http://dx.doi.org/10.1080/03602550802497727

111. Discacciati, J. A. C. \& Oréfice, R. L. - J. Mater. Sci., 42, p.3883 (2007). http://dx.doi.org/10.1007/s10853-006-0459-Z

112. Wilhelm, H. M.; Souza, G. P.; Wypych, F.; Ribeiro, C. \& Sierakowski, M. R. - Met. Mater. Process., 17, p.289 (2005).

113. Valadares, L. F.; Leite, C. A. P. \& Galembeck, F. - Polymer, 47, p.672 (2006). http://dx.doi.org/10.1016/j.polymer.2005.11.062

114. Silva, E. O.; Tavares, M. I. B. \& Nogueira, J. S. - J. Nano Res., 4, p.117 (2009).

115. Bragança, F. C.; Valadares, L. F.; Leite, C. A. P. \& Galembeck, F. Chem. Mater., 19, p.3334 (2007).

Enviado: 11/03/10

Reenviado: $27 / 03 / 11$

Aceito: 04/04/11

DOI: $10.1590 /$ S0104-14282011005000069 